\title{
Oleuropein aglycone: a polyphenol with different targets against amyloid toxicity
}

Manuela Leri ${ }^{\text {a,b }}$, Reiner Oropesa-Nuñez ${ }^{c}$, Claudio Canale ${ }^{c}$, Sara Raimondi ${ }^{\text {d }}$, Sofia Giorgetti ${ }^{\text {dee }}$, Elena Bruzzone ${ }^{\text {a }}$, Vittorio Bellotti ${ }^{\text {de,ff }}$, Massimo Stefani ${ }^{\text {a,f }}$, Monica Bucciantini ${ }^{\text {a,f }}$.

a Department of Biomedical, Experimental and Clinical Sciences 'Mario Serio', University of Florence, Viale Morgagni 5050134 Florence, Italy

b Department of Neuroscience, Psychology, Area of Medicine and Health of the Child of the University of Florence, Viale Pieraccini, 6 - 50139 Florence, Italy

c Department of Physics, University of Genova, via Dodecaneso 33, 16146, Genova, Italy.

d Department of Molecular Medicine, Institute of Biochemistry, University of Pavia, Viale Taramelli 3/B 27100 Pavia, Italy

e Wolfson Drug Discovery Unit, Centre for Amyloidosis and Acute Phase Proteins, Division of Medicine Royal Free Campus, University College London, NW3 2PF London London, UK

f Interuniversity Center for the Study of Neurodegenerative Diseases (CIMN), Florence, Italy

To whom correspondence should be addressed

Monica Bucciantini, monica.bucciantini@unifi.it, Dipartimento di Scienze Biomediche

Sperimentali e Clinche "Mario Serio"- Università degli Studi di Firenze, viale Morgagni 50, 50134 Firenze, Italy 


\begin{abstract}
Background: Many data highlight the benefits of the Mediterranean diet and its main lipid component, extra-virgin olive oil (EVOO). EVOO contains many phenolic compounds that have been found effective against several aging- and lifestyle-related diseases, including neurodegeneration. Oleuropein, a phenolic secoiroid glycoside, is the main polyphenol in the olive oil. It has been reported that the aglycone form of Oleuropein (OleA) interferes in vitro and in vivo with amyloid aggregation of a number of proteins/peptides involved in amyloid, particularly neurodegenerative, diseases avoiding the growth of toxic oligomers and displaying protection against cognitive deterioration.
\end{abstract}

Methods: In this study, we carried out a cellular and biophysical study on the relationships between the effects of OleA on the aggregation and cell interactions of the D76N $\beta 2$-microglobulin (D76N b2m) variant associated with a familial form of systemic amyloidosis with progressive bowel dysfunction and extensive visceral amyloid deposits.

Results: Our results indicate that OleA protection against $\mathrm{D} 76 \mathrm{~N} \mathrm{b2m} \mathrm{cytotoxicity} \mathrm{results} \mathrm{from} \mathrm{i)} \mathrm{a}$ modification of the conformational and biophysical properties of its amyloid fibrils; ii) a modification of the cell bilayer surface properties of exposed cells.

Conclusions: This study reveals that OleA remodels not only D76N b2m aggregates but also the cell membrane interfering with the misfolded proteins-cell membrane association, in most cases an early event triggering amyloid-mediated cytotoxicity.

General significance: The data provided in the present article focus on OleA protection, featuring this polyphenol as a promising plant molecule useful against amyloid diseases.

\title{
Highlights:
}

- Oleuropein aglycone reduces the $\mathrm{D} 76 \mathrm{~N}$ b2 $\mathrm{m} / \mathrm{membrane}$ interaction

- Oleuropein aglycone alters the surface properties of D76N b2m aggregates

- Oleuropein aglycone might be used to counteract b2m- related pathologies

\section{Abbreviation:}

ANS: 8-anilinonaphthalene-1-sulfonic acid

EVOO: extra-virgin olive oil

OleA: Oleuropein aglycone

MD: Mediterranean diet

DMSO: dimethyl sulfoxide

Keywords: Oleuropein aglycone, amyloid, $\beta 2$-microglobulin, D76N variant. 


\section{Introduction}

Systemic amyloidosis include severe, potentially fatal, diseases characterized by multiorgan deposition of insoluble fibrillar polymeric material grown from misfolded proteins. The rarity and the variable involvement of different organs and tissues are often responsible for missed or delayed diagnosis, which makes amyloid diseases a severe clinical challenge. A large wealth of data support the idea that the pathological features of systemic amyloidosis stem primarily from the concurrent presence of amyloid deposits arising from the ordered polymerization of a number of specific proteins and their circulating amyloidogenic precursors [1,2]. Consequently, at the present, the research for new compounds, either synthetic, natural or derived from natural molecular scaffolds, able to interfere with the aggregation of proteins involved in amyloid diseases, is considered a rewarding strategy to develop new therapeutic strategies. Plant polyphenols have been extensively studied for their multiple biological activities, including anti-oxidant, anti-inflammatory, antidiabetic, anti-aggregation and anti-cancer power [3]. These effects have raised the interest in plant polyphenols for their possible use, among others, to prevent tissue deposits of amyloid aggregates and to contrast pathological tissue reactions to the latter [4]. In particular, it has repeatedly been reported that the Mediterranean diet (MD), naturally enriched in plant polyphenols such as resveratrol (contained in red wine) and oleuropein (the main polyphenol in the olive leaves, drupes and oil) and many others is effective against age-related deterioration and improves agingassociated degenerative diseases and neurological deficits [5].

The glycated oleuropein molecule consists of the polyphenol hydroxytyrosol (4-(2hydroxyethyl)benzene-1,2-diol) esterified to both elenolic acid and glucose. Recent research on oleuropein aglycone (OleA), a metabolic product resulting from the action of endogenous $\beta$ glucosidases, has focused on its general ability to remodel and inactivate toxic amyloid oligomers [6]. In particular, OleA interferes with amyloid aggregation of human islet amyloid polypeptide (hIAPP) [7], A $\beta 42$ [8] and transthyretin [9] skipping the appearance of toxic oligomers and/or favouring aggregation into off-pathway aggregates. The behaviour of OleA matches that of many other polyphenols, even though the mechanism of their anti-aggregation effect can differ in different molecules [10]. In vivo studies have confirmed that OleA protects against $\mathrm{A} \beta 42$ aggregation and plaque formation in tissue. These studies were carried out using transgenic animal models of A 342 aggregation and plaque deposition such as C. elegans [11] and the TgCRND8 mouse model [12]. In particular, in the latter, dietary supplementation of OleA strongly improved the cognitive performance with respect to untreated littermates. Such a positive effect matched reduced $\mathrm{A} \beta 42$ levels and plaque deposits in the animal brain together with reduced inflammatory response, increased synaptic function (LTP), and neurogenesis, microglia migration to the plaques 
for phagocytosis and an intense activation of the autophagic flux. These biochemical modifications in OleA-fed mice were traced back, at least in part, to the induction of epigenetic modifications [13]. Finally, a recent study reports that OleA is able to locate at the surface of the cell membrane interacting specifically with negatively charged phospholipids of synthetic lipid bilayers [14], suggesting its effective ability to interact with the cell membrane. This finding is of interest considering that the interaction of OleA with the cell membrane can either interfere with bilayerbinding properties of amyloid species and/or increase the local concentration of the polyphenol itself; the latter can be a particularly relevant effect considering the low amounts of polyphenol intake with a normal diet and its limited bioavailability.

An increasing body of data considers amyloid cytotoxicity as the overall result of a complex interplay between the biochemical and biophysical properties of both amyloid aggregates and the cell membrane they interact with [15-18]. The biochemical, biophysical and mechanistic aspects of this interaction are widely considered an early event of amyloid cytotoxicity and have been the focus of intense research in recent years [15-19]. However, the development of therapeutic strategies has been largely directed to search substances able to interfere with the process of amyloid aggregation [19], whereas the investigation of molecules able to interfere with membrane activity and lipid-binding properties of amyloid species has been addressed only marginally.

In this study, we extended and implemented our previous data on the effects of OleA against the first described naturally occurring structural variant, Asp76Asn (D76N), of human $\beta 2$ microglobulin (b2m) [20-22], a 99 residue protein subunit of the MHC-I produced by all nucleated cells as a 119 residue precursor. b2m displays a $\beta$-sandwich fold typical of the immunoglobulin superfamily and contains seven $\beta$-strands. Normally, once released from the quaternary structure of MHC-I, b2m is cleared from the circulation in the proximal tubules of the kidneys. However, in patients undergoing long-term dialysis, the serum concentration of $\mathrm{b} 2 \mathrm{~m}$ rises from normal levels (1$2 \mathrm{mg} / \mathrm{L}$ ) to as much as $50-70 \mathrm{mg} / \mathrm{L}$. Such a dramatic increase of concentration is believed to be the main cause of $b 2 \mathrm{~m}$ aggregation in these patients. [23]. The pathogenic naturally occurring D76N variant is aggressively fibrillogenic in vitro, forcing a re-evaluation of previously hypothesized mechanisms of b2m fibrillogenesis. In contrast to patients with dialysis-related amyloidosis, all members of the family carrying such mutation had normal circulating concentrations of b2m and normal renal function. Despite the misfolding propensity of the D76N variant and the essential contribution of $\mathrm{b} 2 \mathrm{~m}$ to the structure of the MHC-I complex, none of the heterozygote subjects displayed clinical evidence of immunodeficiency [20]. On the contrary, extensive amyloid deposits of D76N b2m were found in the spleen, liver, heart, salivary glands, and nerves of the homozygotes where, in the absence of adequate therapy, the disease resulted in a fatal outcome [20]. 
We have recently reported that amyloid aggregates of $\mathrm{D} 76 \mathrm{~N} \mathrm{~b} 2 \mathrm{~m}$ interact specifically with the plasma membrane of the exposed cells at the level of GM1, a major lipid rafts component, and this findings was assumed to at the basis, at least in part, of their toxic effects [21]. These data led us to hypothesize that, particularly for $\mathrm{D} 76 \mathrm{~N}$ b2m amyloidosis, inhibiting aggregate-membrane interaction could be a very important way to protect cells against aggregate cytotoxicity thus reducing at least some symptoms of the disease. Accordingly, we extended our previous findings in SH-SY5Y cells by investigating the ability of OleA to interfere with D76N b2m aggregation and aggregate cytotoxicity. We found that OleA reduces D76N b2m cytotoxicity (i.) by favoring the appearance of non-toxic aggregates; (ii.) by decreasing aggregate interaction with the plasma membrane at GM1-enriched sites; (iii.) by enhancing cell resistance against the toxic effects of the aggregates. Our data offer the possibility to validate and optimize the use of OleA to rationally design novel and promising pharmacological tools to be used for prevention and therapy of systemic amyloidosis.

\section{Materials and Methods}

2.1 In vitro aggregation of $\mathbf{D 7 6 N}$ b2m. D76N b2M was expressed and purified as previously reported [22]. The aggregation reaction was initiated starting from the freeze-dried protein. Briefly, the protein was dissolved in PBS (Sigma Aldrich, Saint Louis, MO, USA) and centrifuged for 5 min at $13200 \times \mathrm{g}$. To achieve aggregation, the resulting supernatant was diluted to $20 \mu \mathrm{M}$ concentration; and $300 \mu \mathrm{L}$ of the solution was incubated at $37^{\circ} \mathrm{C}$ in a $1.5 \mathrm{~mL}$ eppendorf tube lying in horizontal position under vigorous shaking. Protein aggregation in the presence of the polyphenol was performed at 1:3 protein:OleA molar ratio. All aggregate concentrations are referred to as monomer concentration.

2.2 Oleuropein deglycosylation. Oleuropein glycoside was purchased from Extrasynthese (Genay, France) and deglycosylated by treatment with almond $\beta$-glycosidase (EC 3.2.1.21, Fluka, SigmaAldrich) as previously described [8]. Briefly, a $10 \mathrm{mM}$ solution of oleuropein glycoside in $310 \mu \mathrm{l}$ of $0.1 \mathrm{M}$ sodium phosphate buffer, $\mathrm{pH}$ 7.0, was incubated with 8.9 I.U. of $\beta$-glucosidase overnight at room temperature. At the end of the incubation, the aglycone (OleA) was precipitated by centrifuging the reaction mixture at $13200 \times \mathrm{g}$ for $10 \mathrm{~min}$. The precipitate was resuspended in dimethyl sulfoxide (DMSO) at $100 \mathrm{mM}$ concentration and batched in aliquots kept frozen and protected from light. Complete oleuropein deglycosylation was confirmed by assaying the glucose released in the supernatant with the Glucose (HK) Assay kit (Sigma-Aldrich, Saint Louis, MO, USA). Each OleA stock was used within the same day once opened. 
2.3 Dynamic Light scattering (DLS). Size distribution analysis of D76N b2m aggregates was carried out at $25^{\circ} \mathrm{C}$ using a Malvern Zetasizer Nano S DLS device (Malvern, Worcestershire, UK) on $20 \mu \mathrm{M}$ protein samples aggregated in the presence or in the absence of OleA. Each sample was centrifuged at $13200 \times \mathrm{g}$ for $30 \mathrm{~min}$ at $4{ }^{\circ} \mathrm{C}$ to spin down large aggregates. Size distributions by intensity and total light-scattering intensity were determined in 10 acquisitions (cell position $4.2 \mathrm{~cm}$, attenuator index 7) of $10 \mathrm{~s}$ each, over a period of $10 \mathrm{~min}$. The reported data are the average of three independent measurements.

2.4 Intrinsic fluorescence measurements. Intrinsic fluorescence Spectra of D76N b2m were collected at $295 \mathrm{~nm}$ excitation before and after adding OleA and the emission intensity was scanned in the 320-430 $\mathrm{nm}$ range. The fluorescence emission spectra were acquired using the Biotek Synergy $1 \mathrm{H}$ plate reader. To perform this experiment we analyzed D76N aggregates obtained in the absence or in the presence of OleA at different times points. We tested also different protein:OleA molar ratios by using OleA at $10 \mu \mathrm{M}$ (1:0.5), $20 \mu \mathrm{M}$ (1:1), $60 \mu \mathrm{M}$ (1:3), $120 \mu \mathrm{M}$ (1:6) and $180 \mu \mathrm{M}(1: 9)$, with respect to $\mathrm{D} 76 \mathrm{~N}(20 \mu \mathrm{M})$. The data were analyzed by subtracting the respective blank spectra (PBS, or OleA/PBS spectrum). Saturation curve, according with Yang JE et al. 2017 [24] was produced by plotting the difference in the intrinsic fluorescence obtained in the absence $\left(\mathrm{I}_{0}\right)$ and presence (I) of OleA as a function of OleA concentration. Dissociation constant $\mathrm{Kd}$ was obtained from the $\mathrm{x}$-intercept of a double-reciprocal plot between $1 /[\mathrm{OleA}]$ and $1 /\left(\mathrm{I}_{0}-\mathrm{I}\right)$ for $\mathrm{x}$ - and $\mathrm{y}$-axis, respectively. Control was performed by OleA addition after D76N aggregates intrinsic fluorescence detection.

2.5 Transmission electron microscopy. TEM analysis was carried out as previously reported [21]. Briefly, $5.0 \mathrm{~mL}$ aliquots of $\mathrm{D} 76 \mathrm{~N} \mathrm{~b} 2 \mathrm{~m}$ were withdrawn from the aggregation mixture at different time intervals, loaded onto a formvar/carbon-coated 400 mesh nickel grids (Agar Scientific, Stansted, UK) and negatively stained with $2.0 \%$ (w/v) uranyl acetate (Sigma-Aldrich, Saint Louis, MO, USA). The grid was air-dried and examined using a JEM 1010 transmission electron microscope at $80 \mathrm{kV}$ excitation voltage.

2.6 Congo Red Assay. The assay was performed as previously described [25] taking into account that amyloid binding induces a characteristic shift from $490 \mathrm{~nm}$ to $540 \mathrm{~nm}$ in the Congo Red (CR) maximum absorbance [26]. In particular, D76N b2m $(20 \mu \mathrm{M})$ aggregation was monitored in the

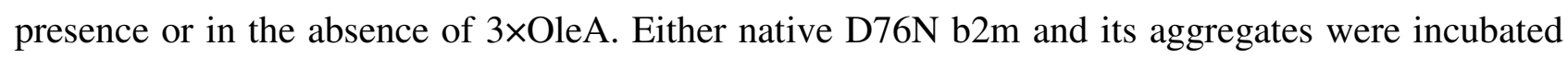
with $5.0 \mathrm{mM}$ sodium phosphate buffer, $\mathrm{pH} 7.0$, containing $150 \mathrm{mM} \mathrm{NaCl}$ and $20 \mu \mathrm{M} \mathrm{CR}$. The CR spectra were recorded in the 400-700 nm region using a Jasco V-630 spectrophotometer. Blank spectra were acquired for $\mathrm{CR}$ alone, in the presence or in the absence of OleA, and subtracted to sample spectra. We performed two types of some controls to asses the OleA interference on CR 
absorbance: i) OleA was added in the same time with $\mathrm{CR}$ on pre-formed aggregates, ii) OleA was added after D76N aggregates CR absorbance detection.

2.7 Thioflavin T fluorescence. Fibrillogenesis of recombinant D76N $\beta 2 \mathrm{~m}(20 \mu \mathrm{M})$ in the absence or in the presence of OleA $(60 \mu \mathrm{M})$ was carried out at $37{ }^{\circ} \mathrm{C}$ in Costar 96-well black-wall plates sealed with a clear sealing film and subjected to $900 \mathrm{rpm}$ double orbital shaking. Bottom fluorescence was recorded at 15 min intervals (FLUOstar Omega, BMG LABTECH). Amyloid growth was monitored by ThT emission at $480 \mathrm{~nm}$ after excitation at $445 \mathrm{~nm}$ in three replicates. We performed two types of some controls to asses the OleA interference on ThT fluorescence: i) OleA was added in the same time with probe on pre-formed aggregates, ii) OleA was added after D76N aggregates ThT fluorescence detection.

2.8 ANS fluorescence. Samples containing aggregating D76N b2M at $20 \mu \mathrm{M}$ concentration in the presence or in the absence of OleA $(60 \mu \mathrm{M})$ were checked for their ability to bind 8anilinonaphthalene-1-sulfonic acid (ANS; Sigma Aldrich, Saint Louis, MO, US). Briefly, $100 \mu \mathrm{L}$ of the aggregation solution were mixed with $500 \mu \mathrm{L}$ of a solution containing $120 \mu \mathrm{M}$ ANS in PBS, then, the fluorescence spectra were recorded in the 400-600 nm range using a Biotek Synergy $1 \mathrm{H}$ plate reader at $370 \mathrm{~nm}$ excitation wavelength. The data were analyzed by subtracting blank spectra consisting of the dye dissolved in PBS in the absence of protein. We performed two types of some controls to asses the OleA interference on ANS fluorescence: i) OleA was added in the same time with probe on pre-formed aggregates, ii) OleA was added after D76N aggregates ANS fluorescence detection.

2.9 Cell culture and cell viability assay. SH-SY5Y cells were cultured at $37{ }^{\circ} \mathrm{C}$ in complete medium (50\% HAM, 50\% DMEM, 10\% foetal bovine serum, $3.0 \mathrm{mM}$ glutamine, 100 units/mL penicillin and $100 \mu \mathrm{g} / \mathrm{mL}$ streptomycin), in a humidified, 5.0\% $\mathrm{CO}_{2}$ incubator. All materials used for cell culture were from Sigma (Sigma Aldrich, Saint Louis, MO, USA). Samples containing aggregating $\mathrm{D} 76 \mathrm{~N}$ b2m were administered to the cells at a final concentration of $5.0 \mu \mathrm{M}$ (monomeric protein concentration). The cytotoxicity of the different forms of D76N aggregates grown at different aggregation times was assessed by the 3-(4,5-dimethylthiazol-2-yl)-2,5diphenyltetrazolium bromide (MTT) (Sigma Aldrich, Saint Louis, MO, USA) reduction inhibition assay based on the protocol described for the first time by Mosmann [27]. In all MTT-experiments, the cells were plated and incubated for $48 \mathrm{~h}$ at a density of 10000 cells/well on 96-well plates in 100 $\mu \mathrm{L}$ culture medium. Then, the cells were treated for $24 \mathrm{~h}$ with $\mathrm{D} 76 \mathrm{~N}$ b2m aggregates $(5.0 \mu \mathrm{M})$ grown in the absence or in the presence of OleA $(15.0 \mu \mathrm{M})$. In some experiments, the cells were treated with $15.0 \mu \mathrm{M}$ OleA for $24 \mathrm{~h}$ before exposure to the aggregates. After exposure, the cells were incubated for $2 \mathrm{~h}$ with $100 \mu \mathrm{L}$ of DMEM without phenol red, containing $0.5 \mathrm{mg} / \mathrm{mL}$ MTT. 
Then, $100 \mu \mathrm{L}$ of cell lysis buffer (20\% SDS, $50 \%$ N,N-dimethylformamide, $\mathrm{pH} 4,7$ ) was added to each well and the samples were incubated at $37{ }^{\circ} \mathrm{C}$ for $2 \mathrm{~h}$ to allow complete cell lysis. Absorbance values of blue formazan were determined at $595 \mathrm{~nm}$ with an automatic plate reader (Bio-Rad, Hercules, Cal). The final absorption values were calculated by averaging three independent measurements of each sample after subtraction of the average of the blank solution (100 $\mu \mathrm{L}$ of MTT solution and $100 \mu \mathrm{L}$ of lysis buffer consisting of $20 \%$ SDS in $50 \% \mathrm{~N}, \mathrm{~N}$-dimethylformamide). All data were expressed as mean \pm standard deviation.

2.10 Confocal Immunofluorescence. Sub-confluent SH-SY5Y cells grown on glass coverslips were treated for $24 \mathrm{~h}$ with the different $\mathrm{D} 76 \mathrm{~N}$ b2m aggregates at a $5.0 \mu \mathrm{M}$ final concentration and then washed with PBS. GM1 labelling at the cell surface was obtained by incubating the cells with $10 \mathrm{ng} / \mathrm{ml}$ CTX-B Alexa488 in complete medium for $10 \mathrm{~min}$ at room temperature. Then, the cells were fixed in $2.0 \%$ buffered paraformaldehyde for $10 \mathrm{~min}$, permeabilized by a cold 1:1 acetone/ethanol solution for $4.0 \mathrm{~min}$ at room temperature and blocked with PBS containing $0.5 \%$ BSA and $0.2 \%$ gelatine. After blockage, the cells were incubated for $1 \mathrm{~h}$ at room temperature with a rabbit polyclonal anti-b2m antibody (Abcam, Cambridge, UK) diluted 1:600 in blocking solution and then washed with PBS for 30 min under stirring. The immunoreaction was revealed by Alexa 568-conjugated anti-rabbit antibody (Molecular Probes, Eugene, Oregon, USA) diluted 1:200 in PBS. Finally, the cells were washed twice in PBS and once in water to remove non-specifically bound Abs. Cell fluorescence was imaged using a confocal Leica TCS SP5 scanning microscope (Leica, Mannheim, Germany) equipped with a HeNe/Ar laser source for fluorescence measurements. The observations were performed using a Leica Plan 7 Apo X63 oil immersion objective suited with optics for DIC acquisition. FRET analysis was performed by adopting the FRET sensitized emission method as previously reported [28].

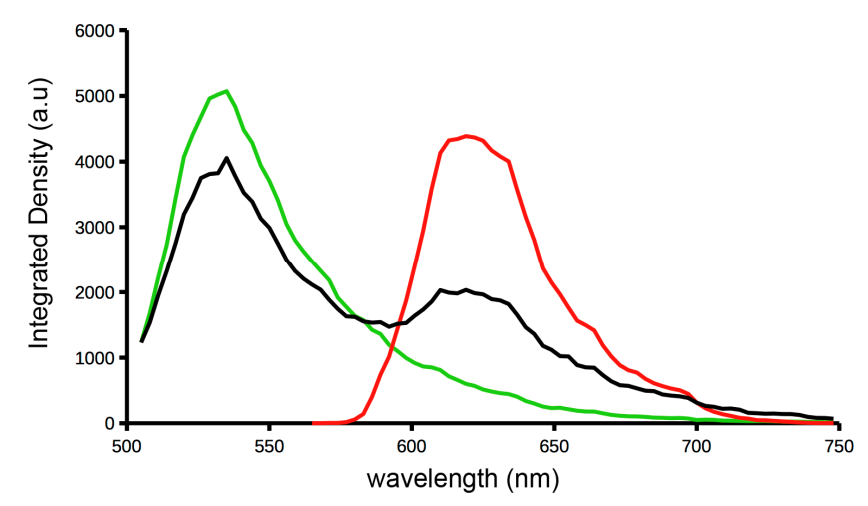

Figure I Spectral analysis of FRET interaction between Alexa-488 fluorophore conjugated with CTX and Alexa 568 fluorophere on immunolabeled b2-m. The emission spectrum of FRET interaction between the two fluorochromes (Alexa-488 fluorophore conjugated with CTX and Alexa 568 fluorophore on immunolabeled $\mathrm{b} 2 \mathrm{~m}$ ) was recorded by exciting the emission maximum of 
the donor with a $458 \mathrm{~nm}$ laser line (black line). The individual emission spectrum of Alexa-488 fluorophore (donor) obtained with $458 \mathrm{~nm}$ laser line is reported as a green line. The individual emission spectrum obtained by exciting Alexa 568 fluorescence (acceptor) with a 543nm laser line is shown as a red line.

2.11 Preparation of supported Lipid Bilayers. 1,2-diOleoyl-sn-glycero-3-phosphocholine (DOPC,18:1), sphingomyelin (SM) (brain, porcine) and ganglioside GM1 (ovine brain, sodium salt) were purchased from Avanti Polar Lipids (Alabama, USA); cholesterol (Chol), chloroform and methanol were from Sigma-Aldrich (Saint Louis, MO, USA). For supported lipid bilayers (SLBs) preparation, lipid mixtures containing DOPC:SM in a 2:1 molar ratio, $1.0 \%$ cholesterol and 5.0\% GM1 were prepared, dissolved in chloroform/methanol (2:1) according to the desired concentration and gently evaporated to dryness under a nitrogen flux. Sample aliquots were maintained overnight under vacuum and resuspended in Milli-Q water to form multilamellar vesicles (MLVs). MLVs were pre-sonicated at $60{ }^{\circ} \mathrm{C}$ for $1 \mathrm{~h}$ and then extruded 11 times through a polycarbonate membrane with $100 \mathrm{~nm}$ pores using a commercial extruder (Avanti Polar Lipids) at $60{ }^{\circ} \mathrm{C}$. Lipid suspensions were cooled at room temperature and the so obtained suspensions of large unilamellar vesicles (LUVs) were diluted 10 folds in Milli-Q water. $40 \mu \mathrm{L}$ of each suspension and $10 \mu \mathrm{L}$ of a $10 \mathrm{mM}$ $\mathrm{CaCl}_{2}$ solution were deposited on a freshly cleaved mica substrate $(1.0 \mathrm{~cm} \times 1.0 \mathrm{~cm})$. To get uniform bilayer coverage, the samples were incubated $15 \mathrm{~min}$ at $60{ }^{\circ} \mathrm{C}$ and then $10 \mathrm{~min}$ at room temperature. Then, the samples were kept at room temperature in a sealed chamber with $100 \%$ relative humidity. SLBs were gently rinsed three times with Milli-Q water to remove excess vesicles from the liquid sub phase before AFM measurements. In some experiments, SLBs, preformed as described in the previous paragraph, were exposed for $30 \mathrm{~min}$ to $15 \mu \mathrm{M}$ OleA and then gently rinsed with MilliQ water to remove the excess of OleA from the solution.

2.12 AFM imaging. AFM images were acquired by using a Nanowizard III (JPK Instruments, Germany) mounted on an Axio Observer D1 (Carl Zeiss, Germany) inverted optical microscope. Sample topography before and after protein aggregate administrations [29] was determined by using the Quantitative Imaging mode (QI - JPK Instruments). Setpoint force was set at $1.0 \mathrm{nN}$ and the length of the acquired force-distance (FD) curves was $80 \mathrm{~nm}$. The tip velocity during acquisition of the FD curves was maintained constant at $20 \mu \mathrm{m} / \mathrm{s}$. V-shaped DNP silicon nitride cantilevers (Bruker, Massachussets, US) with a nominal spring constant $0.24 \mathrm{~N} / \mathrm{m}$ and a typical tip radius of curvature of 20-60 nm were used. The actual spring constant of each cantilever was determined in situ using the thermal noise method. D76N b2m aggregates aged $144 \mathrm{~h}$ grown in the absence or in the presence of OleA at a 1:3 protein:OleA molar ratio were administered to a final concentration of 
$5.0 \mu \mathrm{M}$ to SLBs pre-treated or untreated with OleA. After 30 min of incubation, the SLBs samples were subjected to AFM measurements.

\section{Results}

\subsection{OleA interferes with amyloid aggregation of $\mathrm{D76} \mathrm{N}$ b2m.}

We initially used different biophysical techniques to investigate whether OleA interfered with the aggregation path of D76N b2m previously described (see under Materials and Methods) [22]. DLS analysis and negatively stained TEM showed that the general size and morphology of D76N b2m species obtained until $144 \mathrm{~h}$ of incubation under aggregating conditions were affected by the presence of the polyphenol (Fig. $1 \mathrm{~A}, \mathrm{~B}$ ). We chose the $144 \mathrm{~h}$ time since we previously showed that at that time the sample was populated almost exclusively by amyloid fibrils without any significant presence of precursors [21]. The DLS analysis showed a very broad size distribution of the OleAtreated protein samples with respect to that shown by the protein alone at the same conditions (Fig.1A). TEM images taken at 24 and $96 \mathrm{~h}$ of D76N aggregation in the presence of the polyphenol showed the presence of evident short fibrils radially diverging from densely packed globular clusters, whereas very short fibrils embedded in a diffuse background of small rod-like aggregates were present in the absence of OleA (Fig.1B). After $144 \mathrm{~h}$ of aggregation, long, straight and dispersed $\mathrm{D} 76 \mathrm{~N}$ b2m fibrils were seen, whereas in the presence of OleA the protein formed a dense meshwork of small fibril-like structures buried into amorphous aggregates (Fig.1B). The D76N $\mathrm{b} 2 \mathrm{~m}$ aggregation in the presence or in the absence of the polyphenol was further investigated by the CR (Fig. 1C) and ThT (Fig. 1E) binding assays. The analysis revealed that the protein grown in the presence of OleA did bind CR, as confirmed by the presence of the peak at $570 \mathrm{~nm}$, but to a lesser extent with respect to the protein alone (Fig. 1C). The ThT fluorescence of D76N b2m increased and reached a plateau after $24 \mathrm{~h}$ (Fig.1E), which is indicative of amyloid fibril formation. In this case, OleA did not alter the aggregation kinetics, even though it led to a slight decrease of ThT fluorescence. Interestingly, the ANS assay on D76N b2m aggregates (particularly those aged $24 \mathrm{~h}$ and $144 \mathrm{~h}$ ) grown in the presence of OleA showed a significant reduction of ANS fluorescence, indicating a minor solvent exposure of hydrophobic clusters on aggregates surfaces (Fig.1D). To observe a putative OleA interference with ThT, ANS and Congo Red binding, we performed control measurements where OleA was added to the preformed aggregates just before the experiments and we did not detect any interference (data not shown).

Finally, we investigated the presence of any interaction between OleA and D76N b2m by monitoring the modifications of tryptophan exposure during D76N b2m aggregation in the presence 
or in the absence of the polyphenol. The native protein harbors two Trp residues at positions 60 and 95 that display specific intrinsic fluorescence. $\operatorname{Trp}^{60}$, located in the $\beta$-turn connecting $\beta$-strands D and $\mathrm{E}$, is solvent-exposed, whereas $\operatorname{Trp}^{95}$, at the end of $\beta$-strand $\mathrm{G}$, is partially buried [30]. D76N b2m was incubated with OleA at different molar ratios in aggregating conditions. During aggregation, the wavelength of maximum fluorescence emission was monitored to detect changes in the environments and solvent accessibility of the Trp. The maximum emission wavelength $\left(\lambda_{\max }\right)$ was about $335 \mathrm{~nm}$ in aggregation buffer, as shown in Fig.2C-F. The plot of Trp fluorescence emission spectra in function of aggregation time (Fig. 2A) showed that D76N b2m alone resulted in a time-dependent increase in the emission intensity at $335 \mathrm{~nm}$ (until $50 \mathrm{~h}$ of aggregation) which indicated a Trp increased burial as far as aggregation proceeded in the absence of significant shift in $\lambda_{\max }$. At longer times of aggregation, a decrease of the emission intensity was observed which is indicative of an increase in solvent accessibility of Trp with time (Fig. 2A). The assemblies obtained in the presence of OleA did not show any modification of the shape of the $\operatorname{Trp}$ fluorescence spectrum (Fig. 2C-F) but only a slightly decrease of the intensity with the increase of OleA concentration in aggregating conditions indicating an intrinsic fluorescence quenching due the presence of the polyphenol (Fig. 2A). The quenching effect and the different surface hydrophobicity observed in the presence of OleA were indicative of a surface modification induced by the polyphenol in the aggregated protein, without any kinetic and structural difference as revealed by Congo Red and ThT assay (Fig.1C, E) and CD analysis (data not shown). The specific molecular interaction between $\mathrm{D} 76 \mathrm{~N} \mathrm{b2m}$ and OleA was verified with a hyperbolic curve obtained by plotting the difference in the intrinsic fluorescence observed at $335 \mathrm{~nm}$ in the absence and presence of OleA as a function of OleA concentration (Fig. 2B). A double reciprocal plot of the hyperbolic curve provided a dissociation constant $(\mathrm{Kd})$ of $60 \mu \mathrm{M}$ between EGCG and D76N b2m aggregates (Fig. 2B, inset).

On biophysical set of experiments we had performed all necessary controls to confirm the results obtained when OleA is present during D76N aggregation process. In particular, OleA was added to the preformed aggregates (incubated for 24, 96, 144 hours) just before the experiments (D76N+OleA) and immediately after the CR, ANS and ThT signals recording.

In this control measurement we observed that OleA did not compete with ANS and ThT binding and fluorescence (Fig. 1 insets D-E). Instead the CR absorbance when OleA resulted be reduced, most evident on $144 \mathrm{~h}$ aggregates, but we did not observe a maximum peak shift, confirming that the OleA presences did not interfere on D76N amyloid aggregation (Fig $1 \mathrm{C}$ ). We performed the same controls on D76N aggregates Intrinsic Fluorescence analysis, and as we has saw OleA did not 
quench the intrinsic fluorescence of aggregates when it was added after the reading (Fig. 2 insets CF).

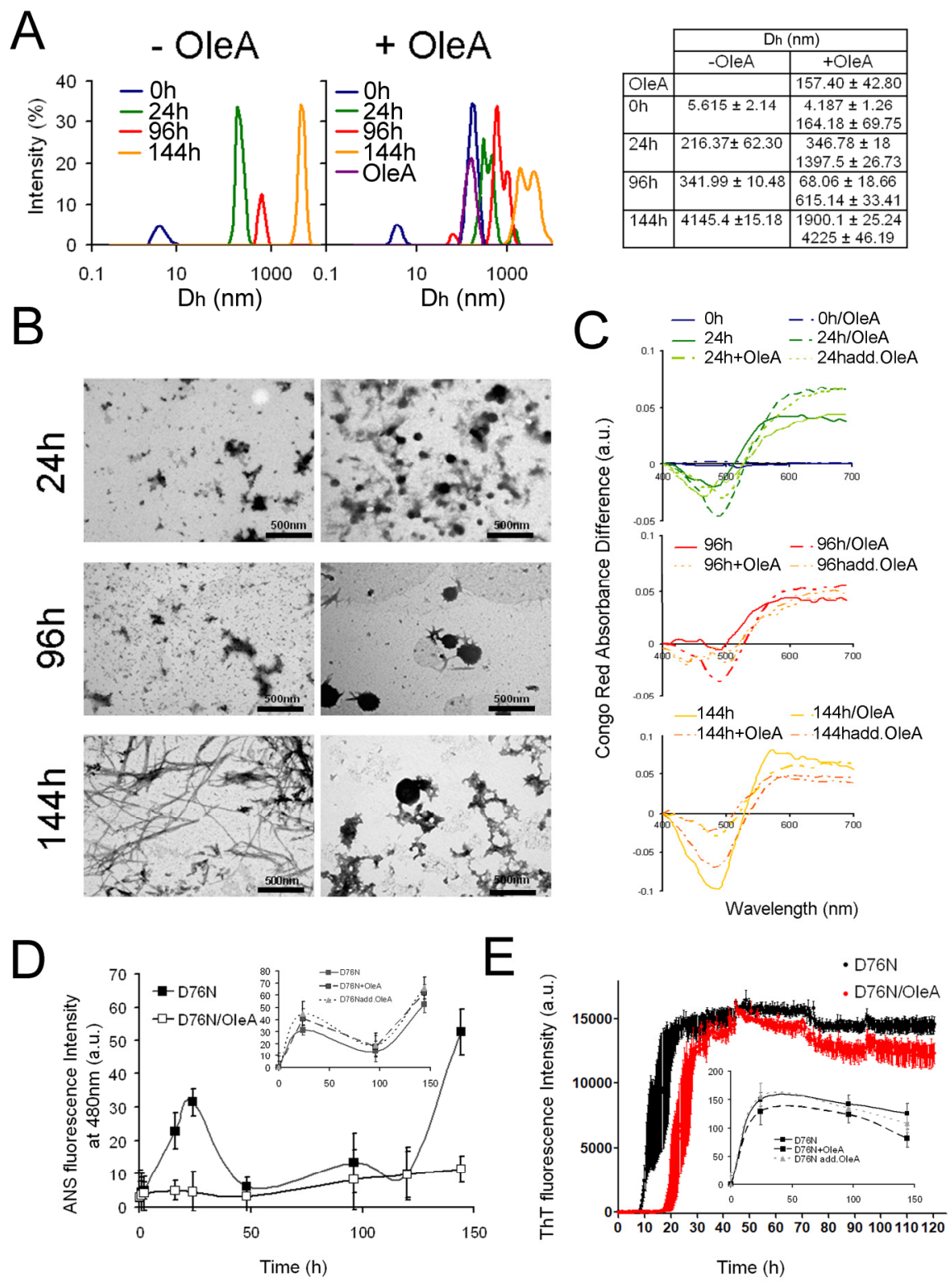

Figure 1-Biophysical characterization of $D 76 \mathrm{~N}$ b2m aggregation in the presence or in the absence of OleA. In panels A and C, color reading is as follows: $0 \mathrm{~h}$ blue, $24 \mathrm{~h}$ green, $96 \mathrm{~h}$ red and $144 \mathrm{~h}$ orange. Control experiments of OleA interference (insets D-E); D76N+OleA: polyphenol and probes were added in same times on pre-formed aggregates; D76Nadd.OleA: polyphenol was added after probes fluorescence detection. (A) Size distributions of D76N b2m alone or in the presence of OleA at four different time intervals. (B-C) TEM images (B) and Congo Red spectra absorption (C). (D) ANS fluorescence Intensity recorded at different times of D76N b2m aggregation in the absence (closed diamond) or in the presence (opened diamond) of OleA (1:3). (E) The time course 
of aggregation of $\mathrm{D} 76 \mathrm{~N} \mathrm{b2m}$, in the absence (black line) or in the presence of OleA (red line; 1:3) monitored by ThT fluorescence emission at $480 \mathrm{~nm}$.
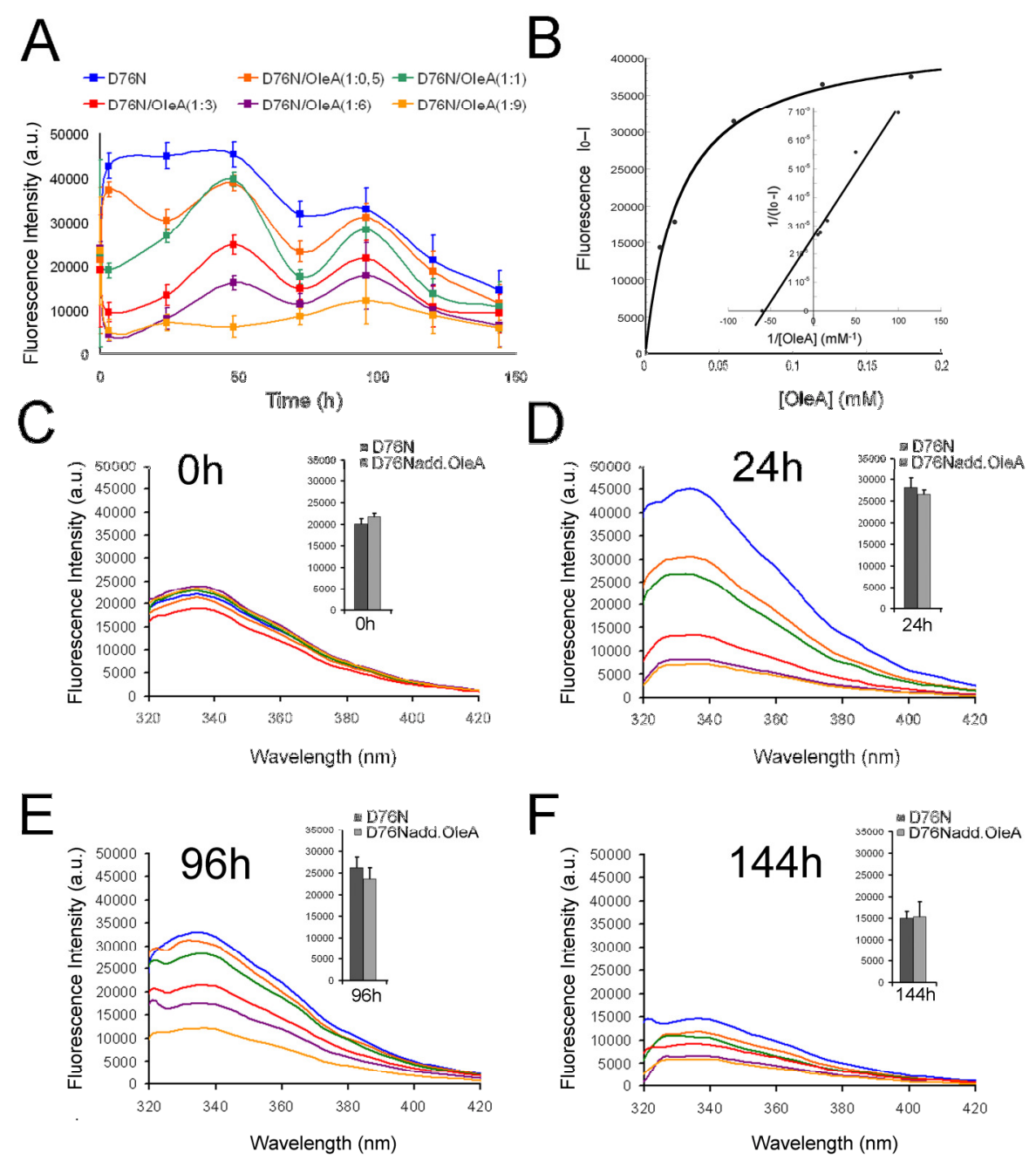

Fig.2 -Effect of OleA on intrinsic fluorescence emission by $\mathrm{D76N}$ b2m. In all panels colors correspond to different D76N:OleA molar ratios: Blue line, protein alone; orange line, 1:0.5; green line, 1:1; red line, 1:3; purple line, 1:6; yellow line, 1:9. A) Intrinsic fluorescence emission intensity as a function of time of $D 76 \mathrm{~N}$ b2m recorded at different aggregation times with different concentrations of OleA. B) Dissociation constant between D76N b2m and OleA assessed with the intrinsic fluorescence of Trp residues of D76N b2m. Differences in the intrinsic fluorescence emitted at $335 \mathrm{~nm}$ in the presence (I) and absence (Io) of OleA were plotted as a function of OleA concentration. Dissociation constant $(K d)$ of $60 \mu \mathrm{M}$ was calculated from a double-reciprocal plot of the saturation curve (Inset). C-F) Spectra of intrinsic fluorescence of D76N b2m obtained at different times B) $0 \mathrm{~h}, C) 24 \mathrm{~h}, \mathrm{D}) 96 \mathrm{~h}$, E) $144 \mathrm{~h}$. To observe a putative OleA interference with Trp fluorescence we recorded spectra before and after addition of OleA (1:3 molar ratio) to preformed 
aggregates and we did not detect any interference (data not shown). Insets) D76N+OleA: control experiments, OleA was added after D76N aggregates intrinsic fluorescence detection.

\subsection{OleA protects against $D 76 \mathrm{~N}$ b2m cytotoxicity.}

It has been reported that the cytotoxicity of amyloidogenic species depends largely on their biophysical surface properties and that the latter influences their reactivity with the cell membrane [31-33]. Once shown that the presence of OleA during aggregation modulates some surface properties of D76N b2m aggregates, we checked by MTT assay whether the cytotoxicity of these samples was the same as that induced by the aggregates grown in the absence of the polyphenol. We used human SH-SY5Y neuroblastoma cells considering that, D76N b2m amyloidosis involves also neural tissue [35] and that these cells had previously been shown to be sensitive to the toxicity of both wt- and D76N b2M aggregates [21, 34]. We found that D76N b2M aggregates grown for 96 $\mathrm{h}, 120 \mathrm{~h}$ or $144 \mathrm{~h}$ were more significantly cytotoxic than those aged $24 \mathrm{~h}$ (Fig 3A), confirming previous data [21]. However, the presence of OleA during protein aggregation affected aggregate toxicity; in fact, we observed a reduction of cytotoxicity from $27.27 \% \pm 11.65 \%$ to $16.93 \% \pm 8.7 \%$ for samples aggregated $96 \mathrm{~h}$, from $41.73 \% \pm 18.21 \%$ to $26 \% \pm 12 \%$ for samples aggregated $120 \mathrm{~h}$ and, more significantly, from $43.84 \pm 10.11 \%$ to $5.9 \pm 13.37 \%$ for $144 \mathrm{~h}$-aged aggregates (Fig. 3A). These data also indicate that the extent of both aggregate toxicity and cytoprotection by the polyphenol increase as far as the aggregation time is prolonged. Moreover, no toxicity was observed when OleA and D76N b2M aggregates were added at the same time to the cells $(144 \mathrm{~h}+\mathrm{OleA})$ or when the cells were pre-treated with OleA for $24 \mathrm{~h}$ prior to exposure to the aggregates (preOleA/144h) (Fig. 3B), suggesting that the polyphenol does not disassemble preformed fibrils with oligomer leakage. Moreover, OleA protection to cells pre-treated for $24 \mathrm{~h}$ persisted even after cell washing with PBS for $30 \mathrm{~min}\left(\mathrm{OleA} 30^{\prime} / 144 \mathrm{~h}\right)$ or $2 \mathrm{~h}(\mathrm{OleA} 2 \mathrm{~h} / 144 \mathrm{~h})$ before exposure to $144 \mathrm{~h}$ aged aggregates (Fig. 3B). In summary, the presence of OleA substantially reduced the toxicity of D76N b2m aggregates in different experimental settings: i) presence of OleA during protein aggregation; ii) cell pre-treatment with OleA before exposure to the aggregates; and iii) cell treatment with OleA and D76N b2M aggregates at the same time (Fig. 3B). These data suggest that OleA interferes not only with the surface aggregates properties but also with the cytotoxic effects of pre-formed aggregates and that OleA-treated cells are more resistant to the aggregates toxic insult.

To better investigate OleA cytoprotection, we used FRET analysis to study aggregate-membrane binding. In agreement with previous results [21], we found that D76N b2m aggregates aged $96 \mathrm{~h}$, 
$120 \mathrm{~h}$ and $144 \mathrm{~h}$ bound to the plasma membrane at, or very close to, the membrane ganglioside GM1 (Fig. 3C column 1,2), a well-known site favouring peptide/protein aggregation [34] and amyloid interaction with the cell membrane [34-36]. When SH-SY5Y cells were treated with D76N b2m aggregates grown in the presence of OleA we observed a lower FRET signal (Fig. 3C column 2). The same FRET signal reduction was detected on SH-SY5Y cells pre-treated with OleA and when the polyphenol was added with $144 \mathrm{~h}$ aggregates (fig. 3D column 2). This finding indicates that the polyphenol interferes with aggregate binding to the membrane modifying the surface properties of either the aggregates and/or the cell membrane (Fig. 3D). The reduced aggregate interaction with the cell membrane in the presence of OleA is likely to contribute to cell protection by the polyphenol.
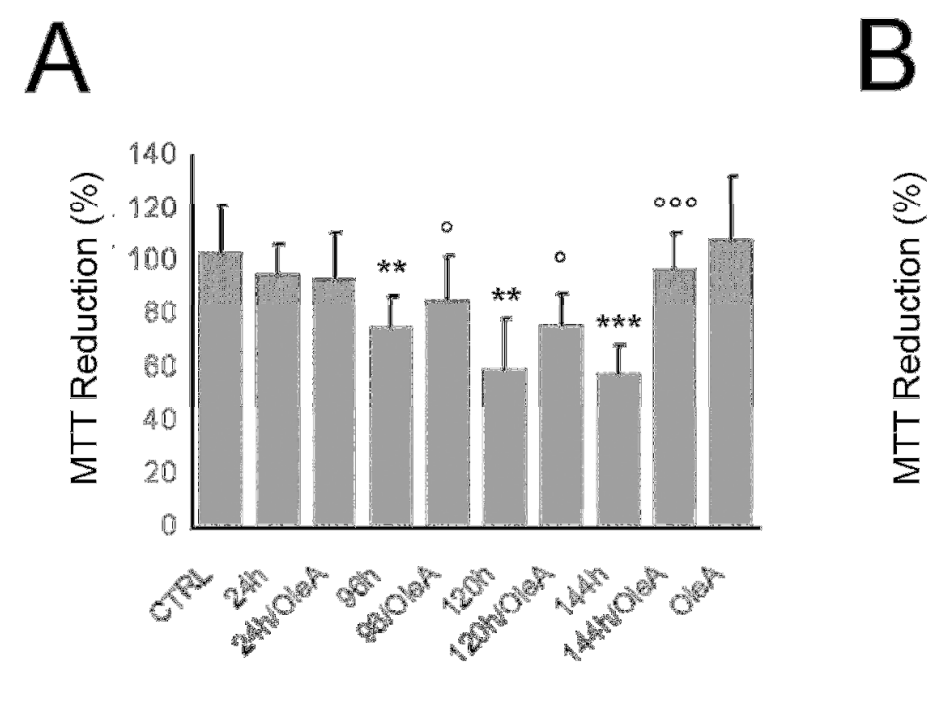
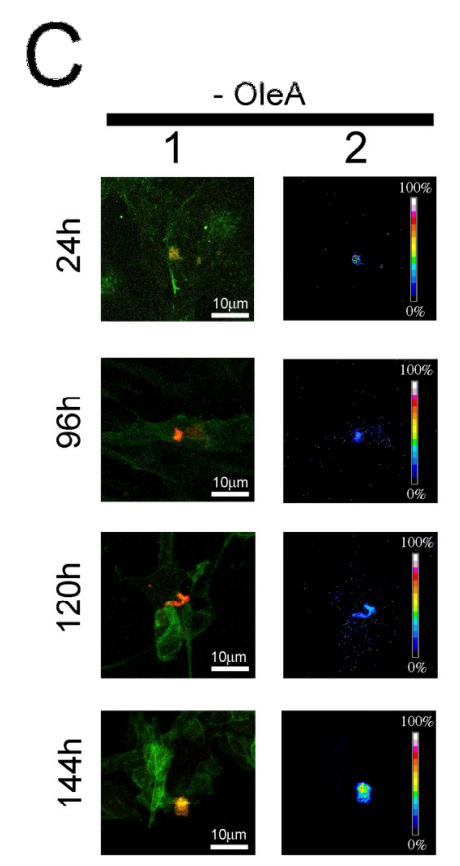
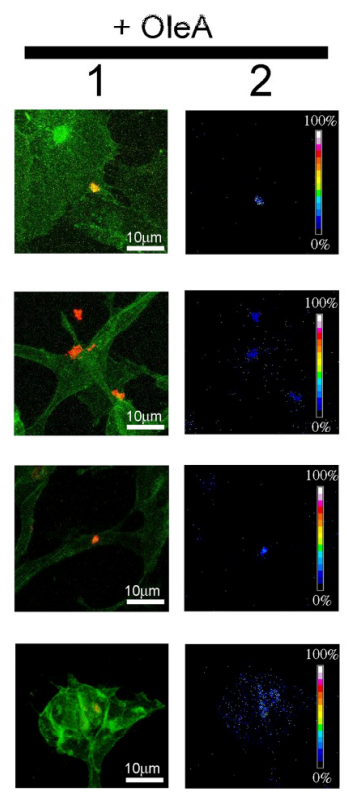
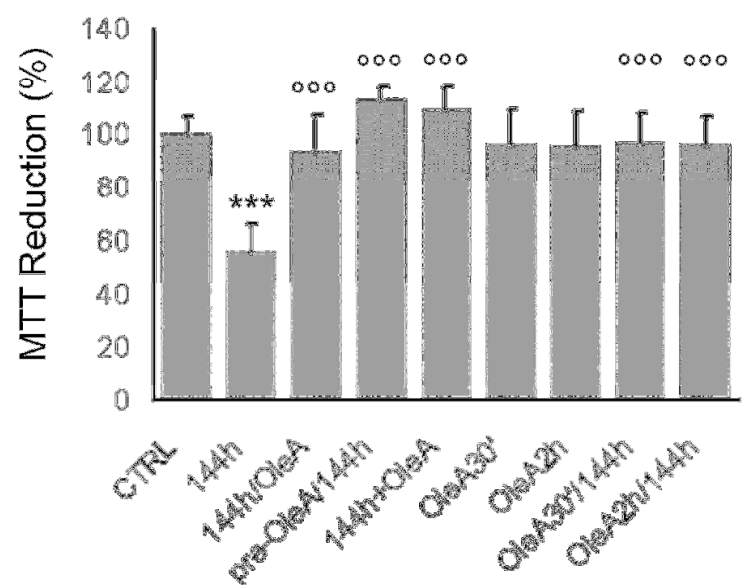

D
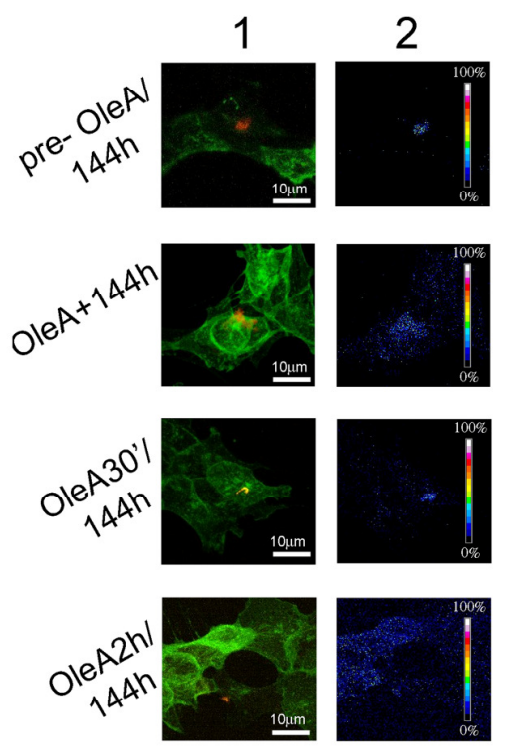
Fig.3 - OleA protects against D76N b2m-induced cytotoxicity. SH-SY5Y cells were treated for $24 \mathrm{~h}$ with $5.0 \mu M$ D76N b2m aggregated for different lengths of time in the absence or in the presence of OleA (1:3 protein:OleA molar ratio). (A-B) The viability of SH-SY5Y cells was assessed by the MTT reduction assay. Error bars indicate the standard deviation of independent experiments carried out in triplicate. $t$-student analysis: * $p<0.05 ; * * p<0.01 ; * * * p<0.001$ vs control; ${ }^{\circ} p<0.05$; ${ }^{\circ}$ $p<0.001$ vs aggregates grown in the absence of OleA. (C-D) OleA interferes with D76N b2m binding to membrane GM1. (C-D) Immunofluorescence (column 1) and FRET analysis (column 2) of the same samples immunostained with Alexa 488-conjugated CTX-B (green fluorescence); protein aggregates were stained with anti-b2m Abs followed by treatment with Alexa 568conjugated anti-rabbit secondary Abs (red fluorescence). (144h/OleA), cells exposed to D76N b2m aggregates aged for $144 \mathrm{~h}$ in the presence of OleA; (pre-OleA/144h): cells pre-treated with OleA for $24 \mathrm{~h}$ and then exposed to D76N b2m aggregates aged $144 \mathrm{~h}$; (OleA30'/144h; OleA2h/144h): cells pre-treated with OleA for $24 \mathrm{~h}$, washed with PBS for 30 min or for $2 \mathrm{~h}$, respectively, to remove OleA from the medium, and then exposed for $24 h$ to D76N b2m aggregates aged $144 h$; $($ OleA+144h): cells treated at the same time with both OleA and D76N b2M aggregates aged $144 \mathrm{~h}$.

\subsection{OleA affects the interaction of D76N b2m with synthetic lipid membranes}

Once assessed that OleA interferes with aggregate binding to the cell membrane and that GM1 is involved in binding, we used GM1-enriched SLBs to better evaluate the effect of OleA on aggregate-membrane interaction using aggregates aged $144 \mathrm{~h}$. SLBs formed on a mica substrate showed segregation of different lipid species in well distinct domains characterized by different levels of order (Fig. 4A). At our conditions, DOPC assembly resulted in SLBs displaying domains with low level of order $\left(\mathrm{L}_{\alpha}\right)$, while the higher level of order $\left(\mathrm{L}_{\beta}\right)$ corresponded to domains enriched in cholesterol, sphingomyelin and GM1, mimicking the lipid composition of membrane raft domains in the cell membrane. The calculated difference in thickness between $\mathrm{L}_{\beta}$ and $\mathrm{L}_{\alpha}$ domains $(\triangle \mathrm{Z})$ was $2.2 \pm 0.2 \mathrm{~nm}$; this value, very reproducible for both untreated SLBs and OleA-treated SLBs (Fig. 4E,G), agrees with previous data [37] and was monitored to evaluate structural perturbations induced by the interaction of $\mathrm{D} 76 \mathrm{~N}$ b2 $\mathrm{m}$ with the bilayer. We found that $144 \mathrm{~h}$-aged aggregates grown in the absence of OleA were accumulating on the GM1-enriched $\mathrm{L}_{\beta}$ domains (Fig. 4B). The presence of aggregates on the ordered phase domains increased the typical thickness of these domains. The height distribution analysis taken from the AFM images of SLBs before and after aggregate administration (Fig. 4E, 4F) indicated that the peak at $2.2 \mathrm{~nm}$, associated to the ordered domains, was replaced by a broader peak at higher height values induced by the accumulation of amyloid material (Fig.4E,F), confirming previous results [20]. However, when the SLBs were 
exposed to $\mathrm{D} 76 \mathrm{~N} \mathrm{b2m} \mathrm{aggregates} \mathrm{grown} \mathrm{in} \mathrm{the} \mathrm{presence} \mathrm{of} \mathrm{OleA,} \mathrm{the} \mathrm{amount} \mathrm{of} \mathrm{aggregates} \mathrm{present}$ on the GM1-enriched phase domains was strongly decreased (Fig.4C) and $\Delta \mathrm{Z}$ was preserved (Fig.4G). A similar reduction of protein interaction with SLBs was found when aggregates preformed in the absence of OleA were added to SLBs pretreated with OleA (see under Materials and Methods). In addition, in this case, although some aggregates accumulated onto $\mathrm{L}_{\beta}$ domains, the SLBs morphology was essentially preserved (Fig.4D, H). A detailed analysis performed on the SLBs before and after OleA treatment confirmed that both the morphology (Fig. 5 A,B) and the elasticity (Fig.5 C-F) of the GM1-enriched membranes were not affected by the treatment with OleA. $\Delta \mathrm{Z}$ was preserved $(2.2 \pm 0.2 \mathrm{~nm})$, also after OleA treatment. These data confirm, in a simplified model and by a different technique, those found with cultured cells pre-treated with OleA reported above.

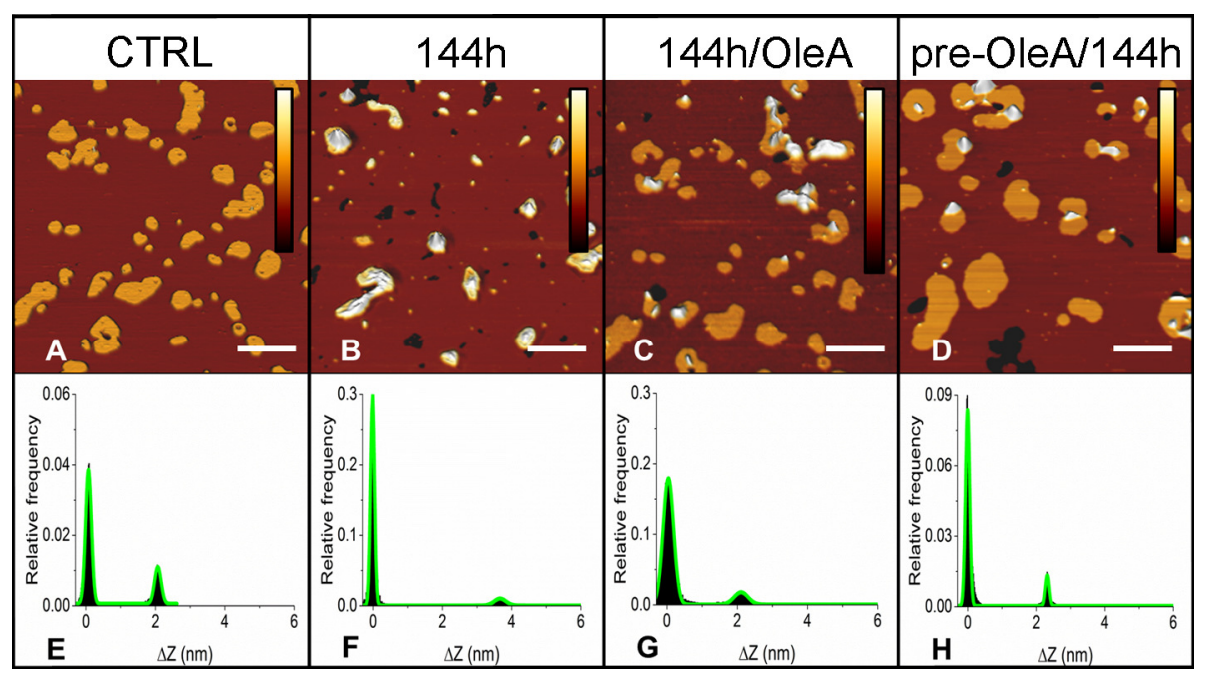

Fig. 4 -AFM analysis. (A) Typical appearance of untreated SLBs in the presence of two different lipid phases. The average $\Delta Z$ for the lipid mixture used in this study was typically around $2.0 \mathrm{~nm}$ [21, 36]. A massive accumulation of protein aggregated for $144 \mathrm{~h}$ was evident on the $L_{\beta}$ phase (B). (C). Aggregate accumulation on the $L_{\beta}$ phase decreased following injection of 144 h-aged aggregates grown in the presence of OleA in a 1:3 protein:OleA molar ratio. (D) The reduced interaction between aggregates and $L_{\beta}$ phase was also evident when toxic 144 h-aged aggregates were injected into SLBs pre-treated for 30 min with $15 \mu M$ OleA. (E-H), Height distributions from data taken from the images displayed in panels $A-D .(F)$ the value of $\triangle Z$ associated to the ordered phase domains increased after administration of 144 h-aged aggregates grown in the absence of OleA. $(G)$ The administration of aggregates grown in the presence of OleA reduced height increase and $\triangle Z$ was preserved. (H), SLBs treatment with OleA was sufficient to protect the membrane 
against aggregate-induced physical modifications; $\Delta Z$ was preserved also in this case. Scale bars: $1.0 \mu \mathrm{m}$; vertical color scale bars: $0-5.0 \mathrm{~nm}$.

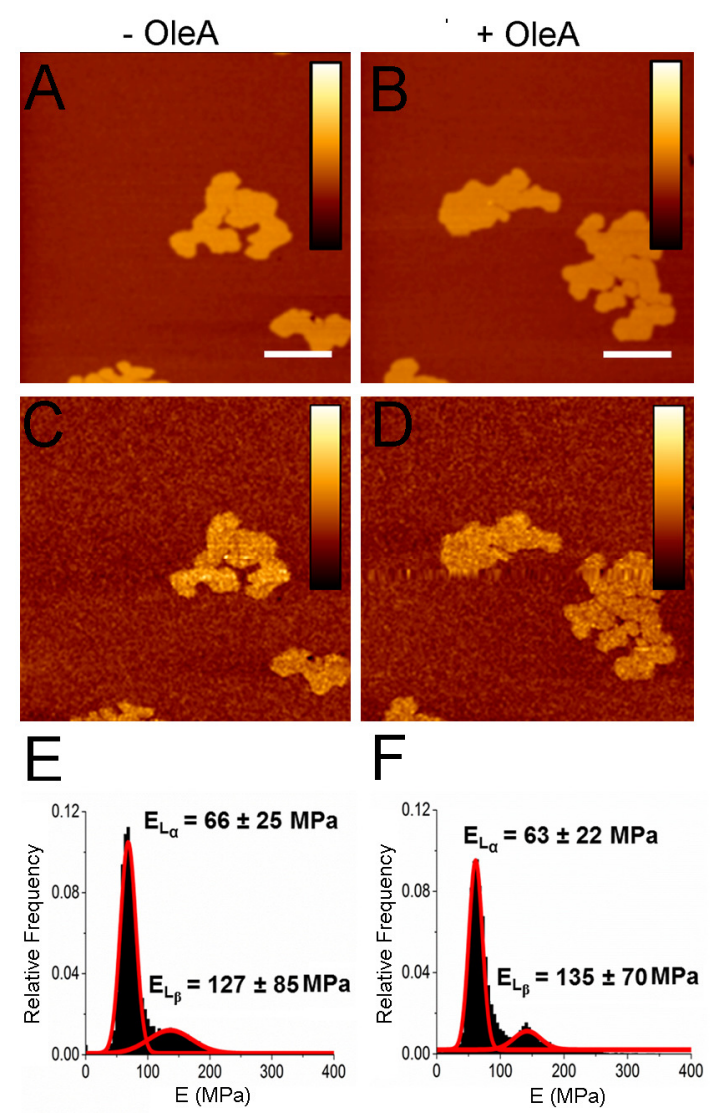

Figure 5. Topographical images $(A, B)$ and elasticity maps $(C, D)$ of $G M 1$ enriched lipid bilayers. The treatment with OleA did not affect the morphology nor the mechanical properties of the membranes. The mean values of the young's modulus of the $L_{\alpha}$ and $L_{\beta}$ domains were not changed after OleA treatment (E,F). Vertical scale bar: A-B 0-8.0 nn; C-D 0-250 MPa.

\section{Discussion}

In systemic amyloidosis the accumulation in tissues and organs of large amounts of proteinaceous deposits may in itself be the primary origin of the clinical symptoms. Anyway, tissue damage and organ dysfunction move forward through mechanisms that are complexes and incompletely understood. The simple explanation of a physical mechanical replacement of parenchymal tissue by amyloid deposits seems to be insufficient. For instance, clinical observations of patients with amyloid light-chain (AL) amyloidosis demonstrated that the reduction by chemotherapy of the amyloidogenic free light chain concentration translated into a reduction of cardiac dysfunction, despite unaltered amyloid deposits in the myocardium [38]. Usually, in the amyloid diseases, it is likely that the impairment of cell function is a direct consequence of the interaction between cellular components and protein aggregates. Although a conspicuous amount of data on cytotoxic effects of amyloid aggregates is presently available, an in depth experimentally-based description of the 
structural features of these assemblies and of the structure-toxicity link is still lacking. The general picture emerging from the findings available in the last few years describes amyloid cytotoxicity as a relative concept rather than an inherent property of amyloid aggregates; in fact, it appears to result from a complex interplay between structural and biophysical features of both the amyloid assemblies and the cell membrane they interact with which determines the final outcome in terms of cell suffering and death [16]. The importance of GM1 as a key binder of amyloid oligomers and aggregates has also been repeatedly reported [17, 39-43].

To date, the research of therapeutic approaches against amyloid toxicity has focused on the inhibition of protein self-assembly and the enhancement of aggregate clearance. In this context, current research aims to identify and describe molecules suitable to counteract protein misfolding, to hinder the appearance of toxic species in the aggregation path and/or to favor the growth of nontoxic assemblies, as well as to interfere with the interaction of the aggregates with the cell membrane and to foster cell resistance against aggregate toxicity. The search for treatments able to reinforce the cell defenses against amyloid cytotoxicity (including those active to maintain correct proteostasis, such as protein synthesis, the ubiquitin-proteasome and autophagy-lysosome pathways), is also actively pursued. Many natural or synthetic molecules that possess some of these properties have been described; among the latter, increasing interest is reserved to plant polyphenols found in several foods particularly enriched in the Mediterranean diet [44]. Resveratrol, curcumin, epigallocathechin-3-gallate (EGCG), quercetin, oleuropein aglycone (OleA) and hydroxytyrosol have been and are being widely studied not only for their well-known antioxidant power but also for their ability to relieve some symptoms of a number of aging-associated degenerative diseases including cancer and neurodegeneration [45]. These studies feature plant polyphenols or their molecular scaffolds as promising chemical tools useful to prevent, to delay and/or to treat many clinical symptoms of these diseases for which, in most cases, an effective therapy is still lacking. This study adds to, and extend, a preceding one [21] by describing the effects of OleA on the aggregation path of $\mathrm{D} 76 \mathrm{~N} \mathrm{b2}$ and on the interaction of the resulting amyloid assemblies with the cell membrane. Our results indicate that the polyphenol does not hinder D76N b2m fibrillization; however, the fibrils obtained in the presence or in the absence of the polyphenol showed distinct conformational and biophysical properties. The change in fluorescence intensity in the $\lambda_{\max }$ observed during protein aggregation in the presence of OleA reflects direct quenching by protein conformational changes resulting from the interaction of the amphipathic polyphenol with hydrophilic and/or hydrophobic regions surrounding the two Trp residues in the protein. The remarkable hydrophobic character of OleA induced conformations with extended hydrophobic surface in protein-OleA complexes favoring the formation of a dense meshwork of small curvilinear 
fibrils. Indeed, the D76N aggregates grown in the absence or in the presence of OleA, showed a very different ability to interact with supported lipid bilayers and with the cell membrane as well as to induce cell suffering. The decreased interaction of the D76N/OleA b2m complex with GM1enriched sites at the cell membrane recorded by FRET analysis suggests that the polyphenol hinders the association of $\mathrm{D} 76 \mathrm{~N}$ b2m fibrils with the cell membrane presumably by binding to the aggregated assemblies.

To shed light on this aspect, we studied in depth the role of OleA in the interaction of D76N b2m aggregates with synthetic lipid membranes by using normal or GM1-enriched lipid bilayers as cell membrane models. Our results confirmed (Figure 4A, E) previous data showing the accumulation of fibrillar D76N b2m assemblies on GM1-enriched L $\beta$ domains [21]. Moreover, our AFM analysis showed that the presence of OleA in the aggregation process interfered with the interaction between fibrillar aggregates and SLBs, with preservation of the morphological and structural features associated with the L $\beta$ domains. Membrane-aggregate interaction was also significantly reduced when D76N b2m fibrillar aggregates grown in the absence of OleA were administered to OleApretreated SLBs. The latter finding indicates that OleA protection against lipid membrane destabilization by amyloids resulted from a modification of the surface properties of both the bilayer and the aggregates induced by OleA during D76N b2m aggregation. This suggestion is supported by recent data indicating that OleA interacts with, and penetrates within, the bilayer in synthetic lipid membranes with different specific locations and interactions depending on membrane lipid composition [13]. It also agrees with the general view reported above which describes amyloid interaction with the cell membrane and cytotoxicity as the result of a complex interplay between aggregates and membrane physical and biochemical properties [16, 46]. We found similar data in OleA-treated cells. In fact, OleA protection to cells pre-treated for $24 \mathrm{~h}$ persisted even after cell washing with PBS for $30 \mathrm{~min}$ or $2 \mathrm{~h}$ before exposure to D76N aggregates. The latter finding suggests OleA persistence within the cell membrane or inside the cytoplasm resulting in biochemical effects making the cell protected against aggregate injury or, alternatively, that the biochemical modifications following cell pre-treatment with OleA persists after polyphenol removal. Taking into account all these data, we suggest that OleA is able to reduce amyloid toxicity by modifying both the aggregate and the membrane biophysical properties; in fact, it did induce conformational and surface changes in $\mathrm{D} 76 \mathrm{~N}$ b2 m aggregates but was also able to interact with the cell membrane with ensuing modification of membrane biochemical/biophysical properties, its biological activity and/or its ability to interact with toxic amyloids. The ability of OleA to affect cell membrane properties was confirmed by a recent investigation; the study reported that rosmarinic acid and black tea extract protect against permeabilization by $\mathrm{A} \beta, \alpha$-synuclein and tau-441 pre- 
fibrillar aggregates of both mitochondria isolated from neuronal SH-SY5Y cells and synthetic vesicles with lipid composition mimicking that of mitochondrial membranes [33, 34].

Recent data suggest the existence of specific interactions between OleA and negatively charged phospholipids in synthetic and cell membranes [14], where they have been reported to represent both nucleation and interaction sites of amyloids [35]. Our data confirm the importance of GM1, a main negatively charged lipid in the cell membrane, as a main site of interaction with D76N b2m fibrils; it also confirms that the presence of OleA in the protein aggregation solution or in the cell culture medium hinders such interaction and, possibly, enhances the cell defenses against the insult provided by the aggregates resulting in reduced cytotoxicity of fibrillar D76 $\mathrm{N} \mathrm{b} 2 \mathrm{~m}$. These pieces of evidence lead to conclude that OleA is protective to our cellular model not only by modifying the structural and biophysical features of the aggregates grown in its presence; in fact, the interaction of OleA with the cell membrane and its reported location inside the bilayer might change both the physical properties and the biological activity of the latter by modulating its functional interaction with other biological molecules and by hindering amyloid binding, a key step of amyloid cytotoxicity.

\section{Conclusion.}

Our data add to, extend and confirm, many others obtained with different amyloidogenic proteins/peptides including amylin, $A \beta$ peptides, transthyretin and wild type $b 2 \mathrm{~m}$, highlighting the interference of OleA with protein aggregation and aggregate cytotoxicity. Taken together, this body of coherent knowledge allows to further generalize the effects of OleA as a molecule with important protective effects against protein amyloid aggregation and some of the molecular and cellular derangements underlying many degenerative diseases with amyloid deposits.

Acknowledgments: This project was supported by Ente Cassa di Risparmio di Firenze $\left(\mathrm{N}^{\circ}\right.$ 2015.1002A2202.3931). M.L. was supported by grant from AIRAlzh Onlus financed by ANCC-

COOP. S.R. was supported by grants from the Cariplo Foundation (Projects 20130964 and 2014 0700), the Telethon Foundation (Grant GG14127), the Italian Ministry of health (Ricerca Finalizzata RF 2013 02355259). 


\section{References}

[1] M. Stefani, Structural features and cytotoxicity of amyloid oligomers: Implications in Alzheimer's disease and other diseases with amyloid deposits. Progress in Neurobiology 99 (2012) 226-245.

[2] M. Stefani and S. Rigacci Beneficial properties of natural phenols: highlight on protection against pathological conditions associated with amyloid aggregation. BioFactors 40 (2014) 482-93.

[3] K.S. Bhullar and H.P. Vasantha Rupasinghe, Polyphenols: Multipotent Therapeutic Agents in Neurodegenerative Diseases. Oxid Med Cell Longev. 2013 (2013) 891748.

[4] B.L. Queen, T.O. Tollefsbol, Polyphenols and aging. Curr. Aging Sci. 3 (2010) 34-42.

[5] N.Y. Scarmeas, M.X. Stern, R. Tang, J.A. Mayeux, J.A. Luchsinger. Mediterranean diet and risk for Alzheimer's disease. Ann. Neurol. 59 (2006) 912-21.

[6] A.R. Ladiwala, M. Mora-Pale, J.C. Lin, S.S. Bale, Z.S. Fishman, J.S. Dordick, P.M. Tessier, Polyphenolic glycosides and aglycones utilize opposing pathways to selectively remodel and inactivate toxic oligomers of amyloid $\beta$. Chembiochem. 12(11) (2011) 1749-58.

[7] S.Rigacci, V. Guidotti, M. Bucciantini, M. Parri, C. Nediani, E. Cerbai, M. Stefani, A. Berti, Oleuropein aglycon prevents cytotoxic amyloid aggregation of human amylin. J Nutr Biochem. 21(8) (2010) 726-35.

[8] S. Rigacci, V. Guidotti, M. Bucciantini, D. Nichino, A. Relini, A. Berti, M. Stefani, A $\beta(1-42)$ aggregates into non-toxic amyloid assemblies in the presence of the natural polyphenol oleuropein aglycon. Curr Alzheimer Res. 8(8) (2011) 841-52.

[9] M. Leri, D. Nosi, A. Natalello, R. Porcari, M. Ramazzotti, F. Chiti, V. Bellotti, S.M. Doglia, M. Stefani, M. Bucciantini, The polyphenol Oleuropein aglycone hinders the growth of toxic transthyretin amyloid assemblies. J. Nutr. Biochem. 30 (2016) 153-66.

[10] A.R. Ladiwala, J.S. Dordick, P.M. Tessier, Aromatic small molecules remodel toxic soluble oligomers of amyloid beta through three independent pathways J Biol Chem. 286(5) (2011) 3209-18.

[11]L. Diomede, S. Rigacci, M. Romeo, M. Stefani, M. Salmona, Oleuropein aglycone protects transgenic $C$. elegans strains expressing $A \beta 42$ by reducing plaque load and motor deficit. PLoS One. 8(3) (2013) e58893.

[12]C. Grossi, S. Rigacci, S. Ambrosini, T. Ed Dami, I. Luccarini, C. Traini, P. Failli, A. Berti, F. Casamenti, M. Stefani, The polyphenol oleuropein aglycone protects TgCRND8 mice against Aß plaque pathology. PLoS One. 8(8) (2013) e 71702.

[13]I. Luccarini, T. Ed Dami, C. Grossi, S. Rigacci, M. Stefani, F. Casamenti, Oleuropein aglycone counteracts A 342 toxicity in the rat brain. Neurosci Lett. 558 (2014) 67-72.

[14] V. Galiano, J. Villalaín, Oleuropein aglycone in lipid bilayer membranes. A molecular dynamics study. J. Biochim Biophys Acta. 1848 (2015) 2849-58.

[15]L.T. Williams, L.C. Serpell, Membrane and surface interactions of Alzheimer's A $\beta$ peptide - insights into the mechanism of cytotoxicity. The FEBS J. 278 (20) (2011) 3905-3917.

[16]C. Cecchi, M. Stefani, The amyloid-cell membrane system. The interplay between the biophysical features of oligomers/fibrils and cell membrane defines amyloid toxicity. Biophysical Chemistry 182 (2013) 30-43.

[17] M. Bucciantini, D. Nosi, M. Forzan, E. Russo, M. Calamai, L. Pieri, L. Formigli, F. Quercioli, S. Soria, F. Pavone, J. Savistchenko, R. Melki, M. Stefani. Toxic effects of amyloid fibrils on cell membranes: the importance of ganglioside GM1. FASEB J. 2012;26(2):818-31.

[18]C. Canale, R. Oropesa-Nun ez, A. Diaspro, S.Dante. Amyloid and membrane complexity: the toxic interplay revealed by AFM. Seminars in Cell and Developmental Biology http://dx.doi.org/10.1016/j.semcdb.2017.08.046 
[19]P. Walsh, G. Vanderlee, J. Yau, J. Campeau, V. L. Sim, C. M. Yip, S. Sharpe, The mechanism of membrane disruption by cytotoxic amyloid oligomers formed by $\operatorname{PrP}(106-$ 126) is dependent on bilayer composition. JBC. 289 (2014) 10419- 10430.

[20]S. Valleix, J.D. Gillmore, F. Bridoux, P.P. Mangione, A. Dogan, B. Nedelec, M. Boimard, G. Touchard, J.M. Goujon, C. Lacombe, P. Lozeron, D. Adams, C. Lacroix, T. Maisonobe, V. Planté-Bordeneuve, J.A. Vrana, J.D. Theis, S. Giorgetti, R. Porcari, S. Ricagno, M. Bolognesi, M. Stoppini, M. Delpech, M.B. Pepys, P.N. Hawkins, V. Bellotti, Hereditary systemic amyloidosis due to Asp76Asn variant beta2-microglobulin. N Engl J Med. 366 (2012) 2276-83.

[21] M. Leri, F. Bemporad, R. Oropesa-Nuñez, C. Canale, M. Calamai, D. Nosi, M. Ramazzotti, S. Giorgetti, F.S. Pavone, V. Bellotti, M. Stefani, M. Bucciantini, Molecular insights into cell toxicity of a novel familial amyloidogenic variant of $\beta 2$-microglobulin. J. Cell. Mol. Med. 20(8) (2016) 1443-56.

[22]P.P. Mangione, G. Esposito, A. Relini, S. Raimondi, R. Porcari, S. Giorgetti, A. Corazza, F. Fogolari, A. Penco, Y. Goto, Y.H. Lee, H. Yagi, C. Cecconi, M.M. Naqvi, J.D. Gillmore, P.N. Hawkins, F. Chiti, R. Rolandi, G.W, Taylor, M.B. Pepys, M. Stoppini, V. Bellotti, Structure, folding dynamics, and amyloidogenesis of D76N $\beta 2$-microglobulin: roles of shear flow, hydrophobic surfaces, and $\alpha$-crystallin. J Biol Chem. 288(43) (2013) 30917-30

[23] T. Eichner, S.E. Radford, Understanding the complex mechanisms of beta2-microglobulin amyloid assembly. FEBS J. 278 (2011) 3868-83.

[24]J.E. Yang, K.Y. Rhoo, S. Lee, J.T. Lee, J.H. Park, G. Bhak, S.R.Paik, EGCG-mediated Protection of the Membrane Disruption and Cytotoxicity Caused by the 'Active Oligomer' of a-Synuclein. Sci Rep. 7(1) (2017) 17945.

[25] M.R. Nilsson. Techniques to study amyloid fibril formation in vitro. Methods. 34(1) (2004) 151-60.

[26]P. Frid, S.V. Anisimov, N. Popovic, Congo red and protein aggregation in neurodegenerative diseases. Brain Res Rev. 53(1) (2007) 135-60.

[27]T. Mosmann, Rapid colorimetric assay for cellular growth and survival: application to proliferation and cytotoxicity assays. J. Immunol. Methods 65(1-2) (1983) 55-63.

[28] D. Nosi, R. Mercatelli, F. Chellini, S. Soria, A. Pini, L. Formigli, F. Quercioli, A molecular imaging analysis of $\mathrm{Cx} 43$ association with Cdo during skeletal myoblast differentiation. J Biophotonics. 6(8) (2013) 612-21.

[29]S. Seghezza, S. Dante, A. Diaspro, C. Canale, High resolution nanomechanical characterization of multi-domain model membranes by fast Force Volume. J. Mol. Recognit. 28(12) (2015) 742-50.

[30] S. Raimondi, N. Barbarini, P. Mangione, G. Esposito, S. Ricagno, M. Bolognesi, I. Zorzoli, L. Marchese, C. Soria, R. Bellazzi, M. Monti, M. Stoppini, M. Stefanelli, P. Magni, V. Bellotti, The two tryptophans of $\beta 2$-microglobulin have distinct roles in function and folding and might represent two independent responses to evolutionary pressure. BMC Evol. Biol. 11 (2011)159.

[31]C. Santambrogio, S. Ricagno, M. Colombo, A. Barbiroli, F. Bonomi, V. Bellotti, M. Bolognesi, R. Grandori, DE-loop mutations affect beta2 microglobulin stability, oligomerization, and the low-pH unfolded form. Protein Sci. 19(7) (2010) 1386-94.

[32] A. Camilleri, C. Zarb, M. Caruana, U. Ostermeier, S. Ghio, T. Högen, F. Schmidt, A. Giese, N. Vassallo. Mitochondrial membrane permeabilisation by amyloid aggregates and protection by polyphenols. Biochim Biophys Acta. 1828(11) (2013) 2532-43.

[33] Y. Ohta, S. Yokoyama, H. Sakai, M. Abe, Membrane properties of binary and ternary systems of ganglioside GM1/dipalmitoylphosphatidylcholine/dioleoylphosphatidylcholine. Colloids Surf B Biointerfaces. 34(3) (2004) 147-53.

[34] M. Bucciantini, S. Rigacci, M. Stefani, Amyloid Aggregation: Role of Biological Membranes and the Aggregate-Membrane System. J Phys Chem Lett. 5(3) (2014) 517-27. 
[35]S. Giorgetti, S. Raimondi, S. Cassinelli, M Bucciantini, M Stefani, G Gregorini, G Albonico, R Moratti, G Montagna, M Stoppini, V Bellotti, beta2-Microglobulin is potentially neurotoxic, but the blood brain barrier is likely to protect the brain from its toxicity. Nephrol Dial Transplant (24) (2009) 1176-81.

[36]E. Evangelisti, R. Cascella, M. Becatti, G. Marrazza, C.M. Dobson, F. Chiti, M. Stefani, C. Cecchi, Binding affinity of amyloid oligomers to cellular membranes is a generic indicator of cellular dysfunction in protein misfolding diseases. Sci Rep. 6 (2016) 32721.

[37] R. Oropesa-Nuñez, S. Seghezza, S. Dante, A. Diaspro, R. Cascella, C. Cecchi, M. Stefani, F. Chiti, C. Canale, Interaction of toxic and non-toxic HypF-N oligomers with lipid bilayers investigated at high resolution with atomic force microscopy. Oncotarget. 7(29) (2016) 44991-45004.

[38]G Merlini, DC Seldin, MA. Gertz, Amyloidosis: Pathogenesis and New Therapeutic Options.. J Clin Oncol 29(14) (2011) 1924-33.

[39]M. Bucciantini, C. Cecchi. Biological membranes as protein aggregation matrices and targets of amyloid toxicity. Methods Mol Biol. 648 (2010) 231-43.

[40] Y Hirakura, B.L. Kagan, Pore formation by beta-2-microglobulin: a mechanism for the pathogenesis of dialysis associated amyloidosis. Amyloid. 8(2) (2001) 94-100.

[41]F. Pellistri, M. Bucciantini, G. Invernizzi, E. Gatta, A. Penco, A.M. Frana, D. Nosi, A. Relini, M.E. Regonesi, A. Gliozzi, P. Tortora, M. Robello, M. Stefani, Different ataxin-3 amyloid aggregates induce intracellular $\mathrm{Ca}(2+)$ deregulation by different mechanisms in cerebellar granule cells. Biochim Biophys Acta. 1833(12) (2013) 3155-3165.

[42]B. Mannini, E. Mulvihill, C. Sgromo, R. Cascella, R. Khodarahmi, M. Ramazzotti, C.M. Dobson, C. Cecchi, F. Chiti, Toxicity of protein oligomers is rationalized by a function combining size and surface hydrophobicity. ACS Chem Biol. 9(10) (2014) 2309-17.

[43] M. Wakabayashi, K. Matsuzaki, Ganglioside-induced amyloid formation by human islet amyloid polypeptide in lipid rafts. FEBS Lett. 583(17) (2009) 2854-2858.

[44]D. Del Rio, A. Rodriguez-Mateos, J.P.E. Spencer, M. Tognolini, G. Borges, and A. Crozier, Dietary (Poly)phenolics in Human Health: Structures, Bioavailability, and Evidence of Protective Effects Against Chronic Diseases. Antioxid Redox Signal. 18(14) (2013) 1818-92.

[45] A.Virmani, L. Pinto, Z. Binienda, S. Ali, Food, Nutrigenomics, and Neurodegeneration Neuroprotection by What You Eat! Mol Neurobiol. 48 (2013) 353 - 362.

[46] M. Hoernke, S. Tassler, B. Koksch, G. Brezesinski, Membrane binding of peptide models for early stages of amyloid formation: Lipid packing counts more than charge. Chem. Phys. Lipids. 198 (2016) 28-38. 